\title{
Atresia coanal congénita unilateral en paciente adulto. Presentación de un caso y revisión bibliográfica sobre vías de abordaje, complicaciones y tratamientos adicionales
}

\author{
Unilateral congenital choanal atresia in adult patient. \\ Case report and bibliographical review about surgical approaches, \\ complications and additional treatments
}

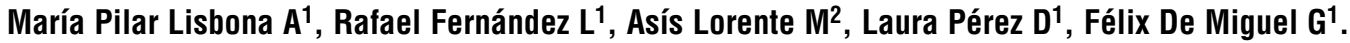

\begin{abstract}
RESUMEN
La atresia de coanas, es una patología poco frecuente, que habitualmente se diagnostica en las edades tempranas. Representa un reto quirúrgico por su alta tendencia a la reestenosis, considerándose en la actualidad de elección el tratamiento endoscópico. Tratamientos como stents posoperatorios o la aplicación de mitomicina $C$ tópica para disminuir la reestenosis son todavía controvertidos, sin que exista consenso. Presentamos el caso de un adulto con atresia de coana unilateral que se reparó vía endoscópica transnasal y se realiza una revisión bibliográfica sobre el estado actual del tratamiento de esta patología.
\end{abstract}

Palabras clave: Atresia de coanas, reparación endoscópica, mitomicina-C tópica.

\section{ABSTRACT}

Choanal atresia is a very rare condition, usually it's diagnosticated in early age. It represents a surgical challenge for the high tendency to restenosis, being actually the endoscopic treatment first option. Other treatments like postoperative stents or topical mitomycin-C in order to avoid restenosis are controversial, and there is a lack of consensus. We present an adult case with unilateral choanal atresia treated by transnasal endoscopic technique and It's carry out a bibliographical review about the current treatment state of this pathology.

Key words: Choanal atresia, endoscopic repair, topical mitomycin-C.

Médico. Servicio de Otorrinolaringología, Hospital Universitario Miguel Servet, Zaragoza, España.

Médico. Servicio de Neurocirugía, Hospital Universitario Miguel Servet, Zaragoza, España. 


\section{INTRODUCCIÓN}

La atresia de coanas es la obstrucción de la apertura nasal posterior, produciéndose una falta de comunicación entre la cavidad nasal y el tracto aerodigestivo. En la mayoría de los casos es de origen congénito, siendo un pequeño porcentaje adquirido, fundamentalmente por complicaciones posradioterapia en carcinomas nasofaríngeos, por trauma quirúrgico o por infecciones de repetición'1.

Descrita por primera vez por J.G. Roderer en 1755, la incidencia se estima en un caso cada 5.000-7.000 nacimientos ${ }^{2}$. La forma unilateral es más frecuente que la bilateral ${ }^{3}$, predomina más en mujeres (2:1), y en el lado derecho ${ }^{2}$. Hasta en $50 \%$ de los casos $^{4}$, se puede asociar con síndromes malformativos como el CHARGE (coloboma, anomalías cardíacas, atresia de coanas, retraso del crecimiento y malformaciones genitales y óticas), el síndrome de Treacher Collins o el síndrome de Tessier. Además existe una incidencia mayor asociada a otro tipo de malformaciones craneofaciales en el síndrome de Down.

Existen cuatro hipótesis principales para explicar esta malformación, que serían: persistencia de la membrana bucofaríngea; adhesiones por localización anormal de tejido mesodérmico; anomalía en la migración de las células de la cresta neural; persistencia de la membrana nasobucal de Hochstetter.

Respecto a la clínica, en los casos bilaterales, al nacimiento se hace evidente una insuficiencia respiratoria nasal, con estridor y episodios de cianosis por desaturaciones, siendo una urgencia con riesgo vital ${ }^{5}$. En los casos de atresia unilateral,

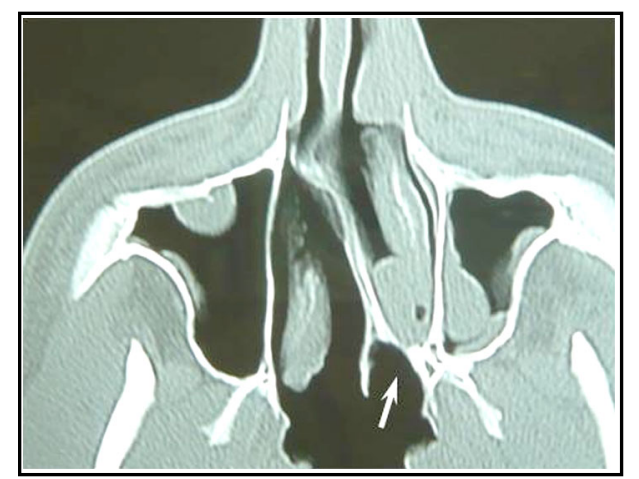

Figura 1. TC axial donde se observa la imperforación de la coana (flecha). la clínica es de obstrucción nasal unilateral, con episodios de rinorrea que se pueden alternar con episodios de infección. Habitualmente el diagnóstico suele ser en los primeros años de la vida. La TC es muy útil en la planificación de la cirugía pues ayuda a identificar la zona atrésica y muestra la anatomía distorsionada por la malformación ${ }^{6}$.

El componente a nivel de la imperforación en $70 \%$ está constituido por un componente osteomembranoso y en el 30\% restante es óseo puro. En el caso de la atresia adquirida suele ser un tejido membranoso fibroso.

\section{CASO CLÍNICO}

Paciente varón de 19 años, que refiere insuficiencia respiratoria nasal de años de evolución. En la exploración inicial de la rinofibroscopía se observa una imperforación a nivel posterior en la coana izquierda y un pólipo nasal. Dado el hallazgo, se estudia el caso con TC, apreciándose un puente óseo con componente de tejido blando que condiciona imperforación en coana izquierda, junto con una desviación del vómer hacia la izquierda (Figuras 1 y 2). Ante el diagnóstico de atresia coanal, se decide realizar intervención quirúrgica transnasal endoscópica. Mediante cirugía endoscópica nasosinusal (CENS), se localiza la zona de la imperforación (Figura 3), encontrándose un tejido mixto osteomembranoso a nivel posterior. Se realiza fresado de la estenosis ósea con resección de la parte membranosa, asociando resección de la parte posterior del vómer y de la lámina perpendicular del etmoides (Figura 4). Además se asocia septoplastía endoscópica

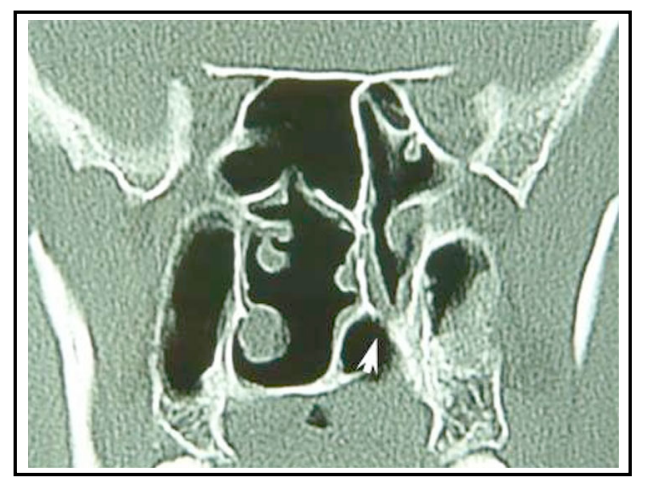

Figura 2. Vista en una TC coronal de la atresia (flecha). 


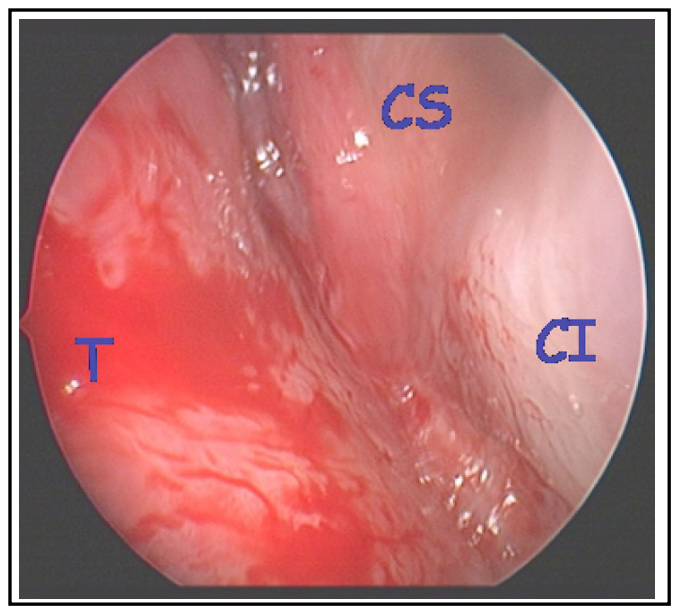

Figura 3. Visión endoscópica de la atresia. T: tabique; CS: cornete superior; $\mathrm{Cl}$ : cornete inferior.

con resección de cresta ósea en parte posterior de la fosa nasal derecha y de zona cartilaginosa en zona anterior de la misma. Finalmente se realizó un colgajo mucoso, que se rebatió sobre la neocoana para evitar una reestenosis, sin que se colocase stent (Figura 5). Se taponó con merocel recubierto con látex, que se mantuvo 2 días. El posoperatorio inmediato transcurrió sin incidencias. Se han realizado sucesivos controles con rinofibroscopio, sin que se haya producido reestenosis a los 19 meses (Figura 6).

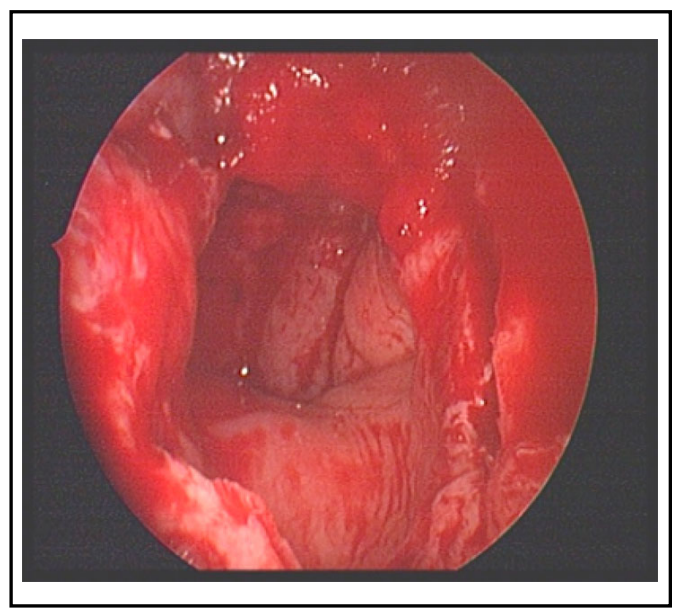

Figura 5. Visión endoscópica de la neocoana recubierta con el colgajo de mucosa.

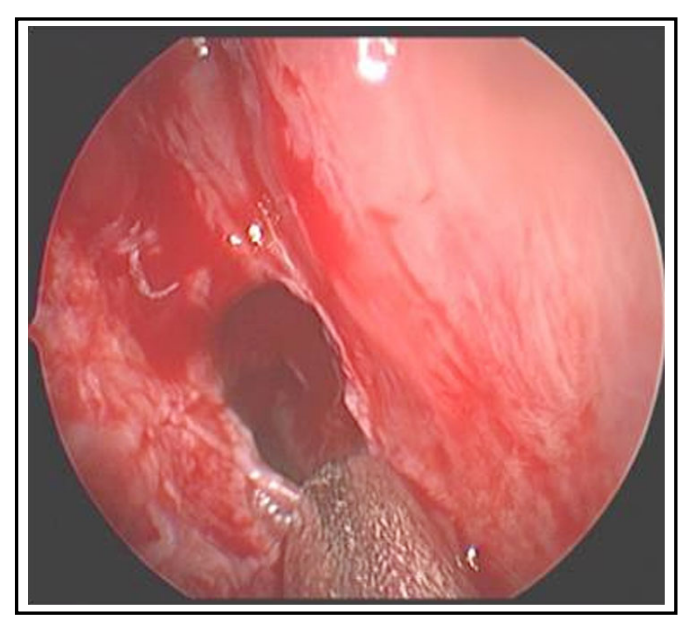

Figura 4. Apertura de la lámina osteomembranosa.

\section{DISCUSIÓN}

Sin duda, la cirugía de la atresia coanal representa un importante reto para los ORL. La principal complicación es la reestenosis, lo que conlleva a un fracaso de la cirugía y puede condicionar la necesidad de una nueva reintervención. La tasa de reestenosis oscila entre $0 \%$ y $85 \%$, si bien en un metaanálisis realizado por Durmaz ${ }^{7}$, que incluía 238 casos, la reestenosis era del $14,7 \%$.

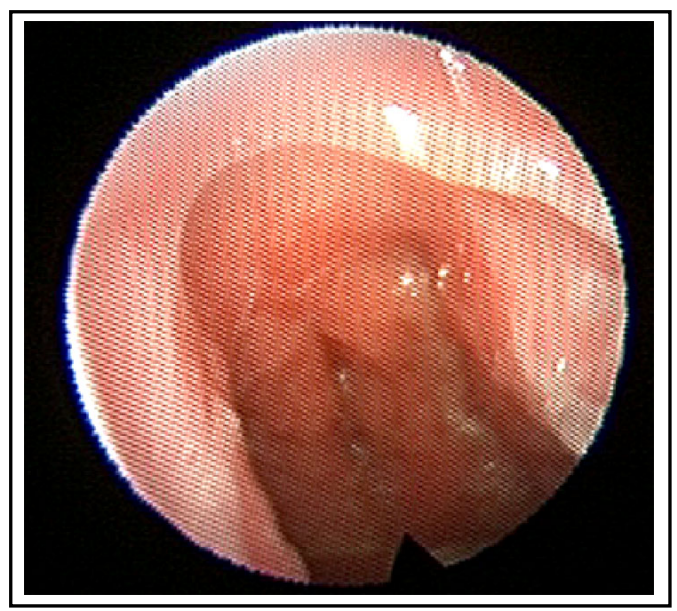

Figura 6. Control endoscópico de la neocoana a los 19 meses, sin apreciarse reestenosis. 
Son muchos los autores que han analizado los factores o procedimientos que pueden ayudar al éxito de la cirugía.

Todos autores están de acuerdo en que el primer paso para afrontar esta cirugía es un correcto estudio del cuadro con fibroendoscopio y un estudio de TC con cortes coronales, axiales y sagitales de corte fino para poder planificar la cirugía con precisión e identificar la zona exacta de placa atrésica. Recientemente se está incorporando el uso de navegadores basados en el TC, que pueden ser de utilidad en determinados casos.

Tras el estudio, hay que decidir qué vía de abordaje se va a realizar. Para esto hay que tener en cuenta factores ${ }^{5}$ como si es uni o bilateral, de naturaleza ósea o membranoso, la edad del paciente y la existencia de otras anomalías craneofaciales que pudieran necesitar tratamiento quirúrgico.

La punción transnasal clásica hoy se considera obsoleta, ya que sólo proporciona alivio temporal, requiriendo ${ }^{2}$ con frecuencia la realización de dilataciones repetidas y con cirugías de revisión.

La vía transpalatal ${ }^{8}$, proporciona una exposición superior a la transnasal, pero es más agresiva, ya que es necesario resecar la parte posterior del paladar óseo, aumenta el tiempo quirúrgico y sangrado y puede ${ }^{1}$ generar complicaciones importantes como fístula palatal, disfunción de la musculatura palatina o trastornos del crecimiento de la arcada alveolar en niños. Esta vía puede ser útil en caso de anomalías asociadas craneofaciales.

La vía transnasal endoscópica se considera la vía de elección. Permite una visión precisa de la zona atrésica, facilitando una resección adecuada de la placa atrésica y de la parte posterior del vómer. El tiempo quirúrgico es corto y el sangrado se minimiza, hecho especialmente importante en niños recién nacidos. Así mismo, permite un buen control de las estructuras vecinas, haciendo que las complicaciones como iatrogenia sobre la base de cráneo, fístulas de LCR, alteración de los centros de crecimiento o lesión de la arteria esfenopalatina sean muy poco frecuentes. Además, facilita un hecho clave que es la preservación de la mucosa con poco traumatismo sobre ella y la posibilidad de realizar con mayor precisión un colgajo mucoso, hecho que se relaciona con unas tasas de éxito quirúrgico más altas. Así mismo, es necesario realizar una resección amplia del vómer $y / 0$ de la parte posterior del tabique, pudiéndose asociar incluso una pequeña resección del receso pterigoide $0^{9-11}$. Las tasas de reestenosis con esta técnica reportadas oscilan entre el 9\%-36\% ${ }^{7}$.

El uso del láser ${ }^{12}$ para la corrección de la atresia es controvertido, ya que si la atresia no es membranosa pura el láser $\left(\mathrm{CO}_{2}, \mathrm{YAG}\right)$ puede tener dificultades para la extirpación de la zona ósea, sin que se consiga una corrección total de la atresia².

Respecto al cuidado posquirúrgico, casi todos los autores recomiendan lavado con suero fisiológico varias veces al día, durante un período de tiempo de semanas o varios meses, e inicialmente asociar corticoides nasales aerosolizados.

El uso de stents posoperatorios en la neocoana es controvertid $0^{13}$. Hengerer ${ }^{2}$, en una de las series clínicas más numerosas, usó stents durante 1-2 semanas de polietileno flexible, con un diámetro de 3,5-4 mm de diámetro extendiéndolos desde la coana a la nasofaringe, sujetándolos para que no dañen el septo. La misión del stent sería la de sujetar el flap mucoso y mantener el calibre de la neocoana. Esta técnica es similar en todos los autores que la usan, sin embargo existe falta de consenso en lo que se refiere a tiempo de mantenimiento o tipo de stent. Otros autores como Schoem $^{14}$, no recomiendan el uso de éstos. Los principales argumentos en contra del stent sería la posibilidad de que produzca reacción a cuerpo extraño con el consiguiente riesgo de fibrosis, y la posibilidad de aumentar el riesgo de infecciones. También podrían comprometer la integridad de la mucosa, factor clave para el éxito quirúrgico, y producir perforaciones septales 0 dañar la columnela. Con el uso de stent, los autores suelen asociar corticoides como el furoato mometasona y antibióticos.

Algunos autores han usado mitomicina $\mathrm{C}$ tópica, para la prevención de la reestenosis ${ }^{11,12,15}$. Sintetizada por el hongo Streptomyces caespitosus es un aminoglucósido que inhibe la proliferación de los fibroblastos por su acción alquilante sobre las cadenas de ADN. Usado como agente antineoplásico inicialmente, actualmente se ha extendido su uso en la prevención de estenosis cicatriciales posquirúrgicas, como en la cirugía del glaucoma, en miringotomías, en dacrocistorri- 
nostomías, en cirugía de drenaje de senos e incluso en cirugía laríngea ${ }^{16}$. De forma similar a como ocurre con el uso de stents, existe disparidad de criterios en la literatura científica sobre el beneficio de la mitomicina ${ }^{17}$. Holland ${ }^{18}$ la usa intraoperatoriamente, teniendo el grupo mitomicina un número menor de dilataciones de revisión estadísticamente significativo. Sin embargo otros autores lo reservan para cuando en las revisiones se ve una tendencia a la reestenosis. La forma más extendida de aplicación es diluir un $\mathrm{ml}$ de una concentración $0,4 \mathrm{mg} / \mathrm{dl}$, y aplicarlo en una torunda durante 3-4 minutos. Efectos adversos graves como el síndrome hemolítico urémico o aplasia medular no se han dado con esta dosificación.

La enfermedad por reflujo gastroesofágico, se correlaciona con una mayor tendencia a la reestenosis, por el posible efecto irritativo. Es más frecuente en niños pequeños, siendo importante asociar tratamiento con antiácidos? .

\section{CONCLUSIONES}

La vía endoscópica transnasal proporciona un acceso y una visión óptima, asociándose en personal entrenado con una menor morbilidad que otras vías como la transpalatal.

La resección amplia del vómer y de la parte posterior del tabique y el manejo exquisito de la mucosa para no traumatizarla son factores claves para el éxito de la cirugía.

Factores como la implantación de stent o el uso de mitomicina son controvertidos, no existiendo consenso en la literatura científica sobre su utilidad.

El lavado nasal con suero fisiológico diario, es recomendado por la mayoría de los autores, pudiendo ser beneficioso.

Por la tendencia tan alta a la reestenosis, es necesario realizar controles frecuentes con rinofibroscopio y realizar desbridamiento precoz si se observa indicios de reestenosis.

\section{BIBLIOGRAFÍA}

1. Wang QY, Wang SQ, Lin S, Chen HH, Lu YY. Transnasal endoscopic repair of acquired pos- terior choanal stenosis and atresia. Chin Med $\mathrm{J}$ (Engl) 2008; 121(12): 1101-4.

2. Hengerer AS, Brickman tM, Jeyakumar A. Choanal atresia: embryologic analysis and evolution of treatment, a 30-year experience. Laryngoscope 2008; 118(5): 862-6.

3. Zuckerman JD, Zapata S, Sobol SE. Single-stage choanal atresia repair in the neonate. Arch Otolaryngol Head Neck Surg 2008; 134(10): 1090-3.

4. Escolan A, Bori Ma, Estropa M, Tisner J, Escolan N, Estropa Torres M. ORL Aragon 2007; 10(1): 20-2.

5. Pardo Romero G, Mogollón Cano-Cortés t, Pando Pinto JM, Trinidad Ruiz G, González Palomino A, Pantoja Hernández C, Blasco Huelva $A$. Endoscopic treatment for choanal atresia. Acta Otorrinolaringol Esp 2007; 58(1): 34-6.

6. Westendorff $\mathrm{C}$, Dammann $\mathrm{F}$, Reinert $\mathrm{S}$, Hoffmann J. Computer-aided surgical treatment of bilateral choanal atresia. J Craniofac Surg 2007; 18(3): 654-60.

7. Durmaz A, Tosun F, Yldrim N, Sahan M, Kvrakdal C, GereK M. Transnasal endoscopic repair of choanal atresia: results of 13 cases and metaanalysis. J Craniofac Surg 2008; 19(5): 1270-4.

8. Ramsden JD, Campisi P, Forte V. Choanal atresia and choanal stenosis. Otolaryngol Clin North Am 2009; 42(2): 339-52.

9. Teissier N, Kaguelidou F, Couloigner V, François M, Van Den Abbeele T. Predictive factors for success after transnasal endoscopic treatment of choanal atresia. Arch Otolaryngol Head Neck Surg 2008; 134(1): 57-61.

10. Josephson GD, Vickery CL, Giles WC, Gross CW. Transnasal endoscopic repair of congenital choanal atresia: long-term results. Arch Otolaryngol Head Neck Surg 1998; 124(5): 537-40.

11. Nour YA, FoAD H. Swinging door flap technique for endoscopic transeptal repair of bilateral choanal atresia. Eur Arch Otorhinolaryngol 2008; 265(11): 1341-7.

12. Ku PK, Tong MC, van Hasselt A. Application of holmium yttrium aluminium garnet (YAG) laser in treatment of acquired posterior choanal atresia following radiotherapy for nasopharyngeal carcinoma. J Laryngol Otol 2007; 121(2): 138-42. 
13. Gosepath J, Santamaria VE, Lippert BM, Mann WJ. Forty-one cases of congenital choanal atresia over 26 years-retrospective analysis of outcome and technique. Rhinology 2007; 45(2): 158-63.

14. Schoem SR. Transnasal endoscopic repair of choanal atresia: why stent? Otolaryngol Head Neck Surg 2004; 131(4): 362-6.

15. Prasad M, Ward RF, April MM, Bent JP, FroeHLICH P. Topical mitomycin as an adjunct to choanal atresia repair. Arch Otolaryngol Head Neck Surg 2002; 128(4): 398-400.

16. Rahbar R, Jones DT, Nuss RC, Roberson DW, Kenna MA, McGill TJ, Healy GB. The role of mitomycin in the prevention and treatment of scar formation in the pediatric aerodigestive tract: friend or foe? Arch Otolaryngol Head Neck Surg 2002; 128(4): 401-6.

17. Rombaux P, de Toeuf C, Hamoir M, Eloy $P$, Bertrand B, Veykemans $F$. Transnasal repair of unilateral choanal atresia. Rhinology 2003; 41(1): 31-6.

18. Holland BW, McGuirt WF JR. Surgical management of choanal atresia: improved outcome using mitomycin. Arch Otolaryngol Head Neck Surg 2001; 127(11): 137580.

Dirección: Dra. María Pilar Lisbona Alquezar Servicio de Otorrinolaringología, Hospital Universitario Miguel Servet 50009 Zaragoza, España

E mail: mplisbona@hotmail.com 